\title{
Scattering Amplitudes from Soft Theorems and Infrared Behavior
}

\author{
Laurentiu Rodina \\ Institut de physique theorique, Universite Paris Saclay, CEA, CNRS, F-91191 Gif-sur-Yvette, France
}

(Received 20 September 2018; revised manuscript received 11 January 2019; published 20 February 2019)

\begin{abstract}
We prove that soft theorems uniquely fix tree-level scattering amplitudes in a wide range of massless theories, including Yang-Mills, gravity, the nonlinear sigma model, Dirac-Born-Infeld, dilaton effective theories, extended theories like the NLSM $\oplus \phi^{3}$ (nonlinear sigma model $\phi^{3}$ ), as well as some higher derivative corrections to these theories. We conjecture the same is true even when imposing more general soft behavior, simply by assuming the existence of soft operators, or by imposing gauge invariance or the Adler zero only up to a finite order in soft expansions. Besides reproducing known amplitudes, this analysis reveals a new higher order correction to the NLSM and two interesting facts: the subleading theorem for the dilaton, and the subsubleading theorem for DBI follow automatically from the more leading theorems. These results provide motivation that asymptotic symmetries contain enough information to fully fix a holographic $S$ matrix.
\end{abstract}

DOI: 10.1103/PhysRevLett.122.071601

Motivation.-Three related concepts are central to quantum gravity: holography, the $S$ matrix, and the black hole information paradox. The holographic principle states that a theory with gravity in the bulk may be described completely by a nongravitational quantum field theory on the boundary. This motivates how Hawking entropy can be proportional to the area of a black hole instead of its volume.

The $S$ matrix on the other hand is the unique local gauge invariant observable of quantum gravity. The naïve merger of quantum mechanics and general relativity leads to tensions with locality and unitarity, and the $S$ matrix should have a formulation that avoids these tensions. This is expected only because the $S$ matrix itself is a naturally holographic object: it describes the $\langle$ in $|$ out $\rangle$ matrix of states measured at asymptotic infinity.

Recently, the holographic nature of the $S$ matrix was made even more concrete by demonstrating the equivalence between asymptotic symmetries and soft theorems [1-5], opening new paths towards finding a holographic dual of flat space-time itself, some of which are reviewed in Ref. [6]. Even more surprisingly, it was proposed that infrared considerations and asymptotic symmetries can have implications for the black hole information paradox [7-9]. This raises an apparently superficial question: how much information can soft particles actually carry?

The goal of this Letter is to take inspiration from the above issues and ask a more well-defined question, in the

Published by the American Physical Society under the terms of the Creative Commons Attribution 4.0 International license. Further distribution of this work must maintain attribution to the author(s) and the published article's title, journal citation, and DOI. Funded by SCOAP. spirit of the $S$-matrix program: how much of an amplitude can be fixed by soft theorems?

The naïve answer is that the low energy (IR) behavior of amplitudes is completely disjointed from the high energy (UV) behavior, so soft particles can only carry some partial information, fixing only the IR part of an amplitude. The following separation seems valid then:

$$
\begin{aligned}
A= & A_{\mathrm{IR}}(\text { Soft theorem satisfying }) \\
& +A_{\mathrm{UV}}(\text { Soft theorem avoiding }) .
\end{aligned}
$$

But, surprisingly, we find that the UV information is not inaccessible via soft theorems-it is simply hidden in several different soft limits. This implies that soft theorems are sufficient to fully fix scattering amplitudes. And in fact, even milder soft behavior can be used instead of the full soft theorems, and still we find that the amplitudes are fixed. This enables us to discover scattering amplitudes starting from soft operators, or what we will call "soft gauge invariance" or "soft Adler zero."

Review of soft theorems.-Soft theorems describe a universal behavior of scattering amplitudes when the energies of one or more massless particles are taken to zero. This limit is taken by rescaling momenta with a soft parameter $p^{\mu} \rightarrow z p^{\mu}$, and taking the $z \rightarrow 0$ limit. The soft theorems then imply a factorization of the following form:

$$
A_{n} \rightarrow\left(z^{\sigma} S^{(0)}+z^{\sigma+1} S^{(1)}+, \ldots\right) A_{n-1},
$$

where the $S_{i}$ are called soft operators and encode symmetries of the theory being considered.

Originally discovered for photons in Ref. [10] and extended to gravitons in Ref. [11], soft theorems have enjoyed a renewed interest, at least in part due to their 
uncovered equivalence to memory effects and asymptotic symmetries [12], and the discovery of a new soft theorem for gravitons [13]. These results subsequently lead to an investigation of soft theorems and asymptotic symmetries for many other theories [14-49]. Soft theorems were shown to follow from considerations of gauge invariance, locality, unitarity, [50,51], shift symmetries in the case of scalar theories [52-54], the CHY formalism [55-60], ambitwistors [61,62], or transmutation operators [63]. Soft theorems for dilaton theories also hinted towards a hidden conformal symmetry in gravity [64].

A different example of surprising soft behavior is known as the Adler zero [65,66], which states that certain scalar amplitudes vanish at leading order in single soft limits:

$$
A_{n} \rightarrow \mathcal{O}(z)
$$

This property has been exploited in the construction of various effective theories: the nonlinear sigma model (NLSM), Dirac-Born-Infeld (DBI), the Galileon [67,68], and special Galileon (see Refs. [55,69] for overviews of these theories).

This was done by constraining the theory space of possible effective theories [70-73], by direct construction using newly enabled recursions $[74,75]$, and most recently through the "soft bootstrap" procedure [76]. This special behavior was also used to rule out possible counterterms in $\mathcal{N}=8$ supergravity $[77,78]$, recently shown to be finite up to 5 loops in $D=4$ [79].

Soft limits as formal Taylor series were also crucial to proving that various scattering amplitudes can be fixed by only three conditions: "weak" locality, gauge invariance or Adler zero, and minimal mass dimension [80,81]. Similarly, in Ref. [82] they were used to argue that there exist unique objects satisfying locality and correct $D$-dimensional BCFW scaling [83-85].

This Letter is a continuation of this previous work, investigating the constraints imposed by locality and various types of soft behavior. In most cases, we find that these properties are even more powerful than gauge invariance or Adler zero, allowing us to also fix the higher mass dimension corrections to various scattering amplitudes. This includes those corresponding to $F^{3}$ or $F^{4}$ operators for Yang-Mills (YM) theory, and similar corrections for general relativity (GR), NLSM, DBI, or conformal dilaton theories. We also directly improve the previous results of Refs. [80,81], by imposing gauge invariance or Adler zero only up to a finite order in soft expansions. Finally, the NLSM (DBI) uniqueness from Adler zero results in the previous work becoming central to proving uniqueness from soft theorems for YM (GR), providing yet a new connection between these theories.

Fixing amplitudes with soft behavior.-The strategy works as follows. First, we pick some theory and a known "seed" amplitude $A_{n-m}$ at $(n-m)$ points, and a corresponding higher point Ansatz $B_{n}\left(p^{k}\right)$, which satisfies the following properties: (1) it is a function only of dot products between $D$-dimensional momenta $p_{i}$ and polarization vectors $e_{i}$ for particles with spin; (2) it is on shell, so it satisfies $n$-particle momentum conservation, as well as $e_{i} p_{i}=0$ and $p_{i} p_{i}=0$; (3) it is local, so its singularities correspond to propagators of $n$-point tree diagrams; and (4) its numerators have mass dimension $k$.

Then we take $m$ particles soft as $p_{i}=z p_{i}, z \rightarrow 0$, obtaining an expansion

$$
B_{n} \rightarrow z^{\sigma} B_{n}^{0}+z^{\sigma+1} B_{n}^{1}+z^{\sigma+2} B_{n}^{2}+, \ldots
$$

Finally we demand that this matches the soft theorems corresponding to our chosen theory (which always only exist up to a finite order $N$ )

$$
B_{n} \rightarrow\left(z^{\sigma} S_{0}+z^{\sigma+1} S_{1}+, \ldots,+z^{N} S_{N}\right) A_{n-m}+, \ldots
$$

This imposes constraints on the free parameters in $B_{n}$, and what we find in many cases is that the Ansatz is fully fixed by this procedure. Only $B_{n}=A_{n}$, the appropriate higher point amplitude, can satisfy all soft theorems.

But the constraints coming from IR behavior can be relaxed beyond what is dictated by soft theorems, and still we find that the UV part is also fixed. Instead of starting with a known amplitude $A_{n-m}$, we can impose the following constraint on two Ansätze $B_{n}$ and $B_{n-m}$ :

$$
B_{n} \rightarrow\left(z^{\sigma} S_{0}+z^{\sigma+1} S_{1}+, \ldots,+z^{N} S_{N}\right) B_{n-m}+, \ldots,
$$

using only the soft operators of some theory as input. We find in many examples that both $B_{n-m}$ and $B_{n}$ are fully fixed, and equal to the amplitudes corresponding to the soft operators used.

Finally, we consider an even more general soft behavior. In the soft expansion [Eq. (4)] we simply require $B_{n}^{0}$ up to $B_{n}^{N}$ to satisfy gauge invariance (for spin theories), or the Adler zero (for pion theories) in the soft particles. Even this very weak condition is apparently sufficient to fix $B_{n}=A_{n}$.

In conclusion, we propose three new constraints pertaining purely to the IR behavior which, together with locality, completely fix various scattering amplitudes: (1) soft theorems, (2) soft operators, and (3) soft gauge invariance or soft Adler zero.

The above results seem to hold for all massless theories which satisfy soft theorems. This includes QED, YangMills theory, gravity, NLSM, DBI, dilaton effective theory [42,86-89], among others. Most such amplitudes are of course already known, but we do discover a novel amplitude: the two extra derivative corrections to the NLSM. Higher corrections to the NLSM were computed in Ref. [90], but those start at four extra derivatives.

In the next section we present the general argument for one of the three claims, namely uniqueness from soft theorems. 
Soft theorem avoiding terms and enhanced soft limits.The argument for uniqueness from soft theorems is straightforward: assume there are two different objects, the amplitude $A_{n}$, and some other function $B_{n}$, satisfying the same soft theorems, which exist up to some order $\mathcal{O}\left(z^{N}\right)$. This implies the difference $B_{n}-A_{n}$ must behave as $\mathcal{O}\left(z^{N+1}\right)$ in all $n$ soft limits. Therefore, after imposing the soft theorems on $B_{n}$, it must take a form

$B_{n} \rightarrow$ [piece satisfying soft theorems $]+\left[\mathcal{O}\left(z^{N+1}\right)\right.$ terms $]$.

The question is then whether such $\mathcal{O}\left(z^{N+1}\right)$ soft theorem avoiding terms can exist at the mass dimension being considered. If these terms do not exist, it follows that $B_{n}=$ $A_{n}$, so there is a unique object satisfying the soft theorems.

Surprisingly, it turns out that, for the most common amplitudes, and even their low lying derivative corrections, this is indeed the case. Their mass dimension is too low to allow any soft theorem avoiding terms.

Proving this is slightly more complicated than simple power counting, because momentum conservation can lead to nontrivial cancellations in soft limits, which contradicts naïve power counting. Various scalar amplitudes do in fact enjoy such an enhanced soft behavior: (1) NLSM, DBI, and special Galileon only exist for even multiplicity, have quartic propagator structure, with numerators of mass dimensions $[n-2],[2 n-4]$, and $[3 n-6]$, with single soft scalings $\mathcal{O}(z), \mathcal{O}\left(z^{2}\right)$, and $\mathcal{O}\left(z^{3}\right)$, respectively; and (2) Galileon vertex: polynomial of mass dimension $(2 n-2)$, with single soft scaling of $\mathcal{O}\left(z^{2}\right)$, and double soft scaling $\mathcal{O}\left(z^{4}\right)$. We will call such objects enjoying enhanced soft limits $A^{\text {enh }}$.

Therefore, depending on the theory, multiplicity, and mass dimension, imposing the soft theorems on an Ansatz $B_{n}$ can lead to one of three outcomes

$$
\begin{gathered}
B_{n}=A_{n}, \\
B_{n}=\operatorname{IR}\left[A_{n}\right]+f(e, 1 / K) A^{\mathrm{enh}},
\end{gathered}
$$

or

$$
B_{n}=\operatorname{IR}\left[A_{n}\right]+[\text { trivially scaling objects }] \text {, }
$$

where $\operatorname{IR}\left[A_{n}\right]$ is the IR piece of $A_{n}$ that is under the control of soft theorems, and $f(e, 1 / K)$ can be some function of polarization vectors and propagators.

In the following sections we investigate several theories in detail.

Yang-Mills theory.-Color-stripped Yang-Mills amplitudes satisfy single soft theorems [14]

$$
A_{n} \rightarrow\left(\frac{1}{z} S_{0}+z^{0} S_{1}\right) A_{n-1}+\mathcal{O}(z)
$$

where the leading soft factor is

$$
S_{0}=\frac{e_{n} p_{1}}{p_{n} p_{1}}-\frac{e_{n} p_{n-1}}{p_{n} p_{n-1}}
$$

which hold even for amplitudes with higher derivative corrections. These correspond to higher mass dimension operators in the general effective Lagrangian [91-93]:

$L=F^{2}+a_{0} F^{3}+a_{1} F_{1}^{4}+a_{2} F_{2}^{4}+a_{3} F_{3}^{4}+a_{4} F_{4}^{4}+, \ldots$,

where the $F_{i}^{4}$ operators represent the different possible contractions of four field strengths.

Our goal is to obtain the various amplitudes by starting from a local Ansatz, $B_{n}\left(p^{k}\right)$. Since we are after YM amplitudes, the Ansatz must be multilinear in $n$ polarization vectors, its poles must correspond to propagators of planar, ordered, graphs with trivalent vertices (such as in Fig. 1), its mass dimension must match, so we fix $k=n-2+\kappa$ powers of momenta in the numerators. We introduce $\kappa$ to keep track of the extra number of derivatives in the operator considered: $\kappa=0$ corresponds to the usual YM amplitude; $\kappa=2$ corresponds to the amplitude with an $F^{3}$ operator insertion; for $\kappa=4$ there are five different amplitudes, corresponding to an $\left(F^{3}\right)^{2}$ or an $F^{4}$-type operator insertion.

Soft theorems: To illustrate the procedure, we first consider the $n=5$ case, which can be checked explicitly, and then prove the statement for general $n$.

The $\kappa=0$ Ansatz, $B_{5}\left(p^{3}\right)$ contains five diagrams, with around 500 terms per diagram. For example, one such term corresponding to the diagram in Fig. 1 is this:

$$
B_{5}\left(p^{3}\right)=a_{1} \frac{e_{5} p_{1} e_{1} e_{2} e_{3} e_{4} p_{1} p_{2}}{\left(p_{1}+p_{2}\right)^{2}\left(p_{1}+p_{2}+p_{3}\right)^{2}}+, \ldots
$$

where $a_{1}$ is one of the free parameters of the Ansatz. Next we impose the following soft theorems:

$B_{5}\left(p^{3}\right) \rightarrow \frac{1}{z} B_{5}^{-1}+z^{0} B_{5}^{0}+, \ldots,=\left(\frac{1}{z} S_{0}+z^{0} S_{1}\right) A_{4}+, \ldots$,

by matching

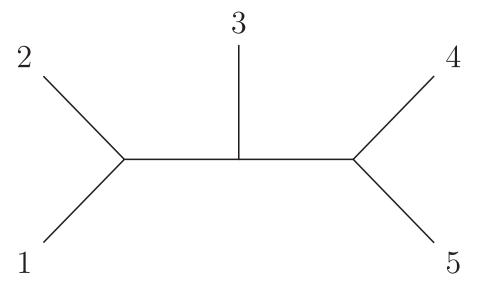

FIG. 1. Cubic five-point tree diagram in Yang-Mills theory. 


$$
B_{5}^{-1}=S_{0} A_{4}, \quad B_{5}^{0}=S_{1} A_{4} .
$$

This leads to a system of equations for the coefficients $a_{i}$, which turns out to have a unique solution: $A_{5}$, the $n=5$ Yang-Mills amplitude.

We can do the same, starting with a higher mass dimension Ansatz, $\kappa=2$. Compared to a term like Eq. (14), the numerators will in this case have two extra powers of momenta. Imposing for each particle:

$$
B_{5}\left(p^{5}\right) \rightarrow\left(\frac{1}{z} S_{0}+z^{0} S_{1}\right) A_{4}^{F^{3}},
$$

where $A_{4}^{F^{3}}$ is the known four-point amplitude with an $F^{3}$ operator insertion. Again we find a unique solution: $B_{5}\left(p^{5}\right)=A_{5}^{F^{3}}$, the corresponding five-point amplitude with an $F^{3}$ insertion.

We can go to an even higher mass dimension at $\kappa=4$, using the known lower point amplitudes $A^{\kappa=4} \equiv a_{1} A^{\left(F^{3}\right)^{2}}+$ $a_{2} A^{F_{1}^{4}}+a_{3} A^{F_{2}^{4}}+a_{4} A^{F_{3}^{4}}+a_{5} A^{F_{4}^{4}}$, and imposing the following:

$$
B_{5}\left(p^{7}\right) \rightarrow\left(\frac{1}{z} S_{0}+z^{0} S_{1}\right) A_{4}^{\kappa=4} .
$$

There are five solutions, as expected: $B_{5}\left(p^{7}\right)=A_{5}^{\kappa=4}$.

Increasing the mass dimension to $\kappa=6$, terms like

$$
\frac{\left(\text { e.ee.ee. } p_{5}\right)\left(p_{1} \cdot p_{2} p_{3} \cdot p_{4}\right)^{2}}{p_{1} \cdot p_{2} p_{3} \cdot p_{4}} \text {. }
$$

have manifest $\mathcal{O}(z)$ scaling and escape all soft theorems, so, in this case, only the IR part of the amplitude is fixed.

Next we prove rigorously in which cases soft theorems fully determine the YM amplitudes.

Proof.-The proof follows immediately from counting arguments and the uniqueness results in Refs. [80,81]. To figure out in which of the cases [Eqs. (8)-(10)] we land, we have to compute the numerator mass dimension required to form $A^{\text {enh }}$ or the trivially scaling objects, both of which must scale as $\mathcal{O}(z)$ in all $n$ soft limits.

Ignoring the denominators, forming a term which trivially satisfies this scaling requires numerators of mass dimension $n$. For $n>4$, the locality of the cubic propagator structure enforces the existence of at least two 2-particle poles per term, which are singular in four different soft limits. Therefore $n>4$ numerators must have mass dimension at least $[n+4]$ to also cancel the soft scaling of the singular poles. At $n=4$ numerators of mass dimension 4 are sufficient to obtain trivial $\mathcal{O}(z)$ objects.

Because the Ansatz has an ordered propagator structure, the only compatible and relevant $A^{\text {enh }}$ are NLSM amplitudes. The polarization vectors in the YMAnsatz can be factored out and ignored. To form quartic NLSM amplitudes [with $(n / 2-2)$ propagators per term], from a cubic YM Ansatz [with $(n-3)$ propagators per term] all the extra propagators must be canceled. This requires numerators of mass dimension $[n-2+2(n-3)-2(n / 2-2)]=[2 n-4]$.

Now the uniqueness argument proves that all Ansätze $B_{n}\left(p^{n-2+\kappa}\right)$ are fixed as long as

$$
n-2+\kappa<\min (n+4,2 n-4),
$$

for $n \geq 5$.

Soft operators: We can replace the known lower point amplitudes with another local Ansatz, and impose

$$
B_{5}\left(p^{3}\right) \rightarrow\left(\frac{1}{z} S_{0}+z^{0} S_{1}\right) B_{4}\left(p^{2}\right)
$$

for each particle. It can be checked the solution is unique: we find $B_{5}=A_{5}$ and $B_{4}=A_{4}$, the expected YM amplitudes.

This is still true for $\kappa=2$, where we find $B_{5}=A_{5}^{F^{3}}$, and also $B_{4}=A_{4}^{F^{3}}$. However, with $\kappa=4$, besides the usual amplitudes, we also find an extra nongauge invariant solution.

As a result of these observations, we conjecture that for general $n$ soft operators fully fix the $\kappa=0,2$ amplitudes, while $\kappa=4$ amplitudes are fixed up to possible extra nonphysical solutions.

Soft gauge invariance: We now relax the imposed IR behavior even further, and demand that in the various soft expansions:

$$
B_{5}\left(p^{3}\right) \underset{p_{i} \rightarrow 0}{\rightarrow} \frac{1}{z} B_{5 ; i}^{-1}+z^{0} B_{5 ; i}^{0}+, \ldots,
$$

the functions $B_{5 ; i}^{-1}$ and $B_{5 ; i}^{0}$ are gauge invariant in particle $i$. We find a unique solution $B_{5}=A_{5}$, and conjecture that this remains true at higher multiplicity, for $\kappa=0$.

Gravity.-Compared to Yang-Mills theory, gravity amplitudes satisfy one extra soft theorem [13],

$$
A_{n} \rightarrow\left(\frac{1}{z} S_{0}+z^{0} S_{1}+z^{1} S_{2}\right) A_{n-1}+\mathcal{O}\left(z^{2}\right),
$$

have unordered cubic propagators, are multilinear in polarization tensors $e_{i}^{\mu \nu} \equiv e_{i}^{\mu} e_{i}^{\nu}$, and have numerators of mass dimension $[2 n-4+\kappa]$. Again we use $\kappa$ to label different amplitudes, with $\kappa=0$ the usual GR amplitudes, $\kappa=2$ the $R^{2}$ operator insertion, and so on.

We can use identical reasons as for YM to prove that GR amplitudes are fixed by soft theorems. The soft theorem avoiding terms in this case must scale as $\mathcal{O}\left(z^{2}\right)$ in all soft limits, so $A^{\text {enh }}$ in this case can be either DBI amplitudes or Galileon vertices. Forming DBI amplitudes from a cubic Ansatz requires mass dimension [3n-6] numerators, while the Galileon requires $[2 n-2]$. Both are always smaller than the trivially scaling object, which requires $[2 n+4]$, due to the two singular two-particle poles at $n>4$. This implies that soft theorems fully fix GR amplitudes if 


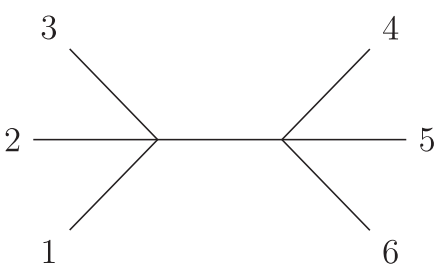

FIG. 2. Quartic six-point tree diagram in NLSM.

$$
2 n-4+\kappa<\min (3 n-6,2 n-2),
$$

for $n \geq 5$.

Finally, we conjecture that soft operators fix the $\kappa=0,2$ cases, and the $\kappa=4$ case up to possible nongauge invariant terms. We also conjecture that soft gauge invariance fixes the $\kappa=0$ case.

NLSM.-Besides the single soft theorems known as the Adler zero $[65,69,94]$, the NLSM also satisfies double soft theorems $[57,95]$ :

$$
A_{n} \rightarrow\left(z^{0} S_{0}+z S_{1}\right) A_{n-2}+\mathcal{O}\left(z^{2}\right) .
$$

In this case soft theorem avoiding terms must scale as $\mathcal{O}\left(z^{2}\right)$ in double soft limits. The starting Ansatz for the NLSM, $B_{n}\left(p^{n-2+\kappa}\right)$, has poles corresponding to propagators of ordered, quartic diagrams, like in Fig. 2. Three-particle poles are singular in double soft limits, and there are a minimum of two three-particle poles per term for $n \geq 8$. Then the trivially scaling object then requires $[n+4]$ mass dimension numerators. There is no relevant $A^{\text {enh }}$ for NLSM amplitudes.

Therefore NLSM amplitudes are fixed for

$$
n-2+\kappa<n+4,
$$

for $n \geq 8$.

To our knowledge a $\kappa=2$ correction has not yet been computed, but the soft operator approach allows us to check if such an amplitude exists.

Soft operators: By imposing

$$
B_{6} \rightarrow\left(z^{0} S_{0}+z S_{1}\right) B_{4},
$$

it can be checked that both Ansätze are fully fixed for $\kappa=0,2$, and 4 . The missing four-point amplitude at $\kappa=2$ is given by:

$$
A_{4}^{\kappa=2}=s_{12} s_{14},
$$

but strangely does not satisfy Bern-Carrasco-Johansson relations [90,96-99].

As before, we conjecture that soft operators fix the higher multiplicity amplitudes.

Soft Adler zero: We can also impose a "soft Adler zero" to obtain the NLSM amplitude. At six points, taking a formal double limit in say particles 5 and 6 :

$$
B_{6} \rightarrow \frac{1}{z} B_{6}^{-1}+z^{0} B_{6}^{0}+z B_{6}^{1}+, \ldots,
$$

we now impose that the three terms above have $\mathcal{O}(z)$ behavior when taking particles 5 and 6 separately soft. Repeating the procedure for the other particles, we find that $B_{6}$ must be the $\kappa=0$ NLSM amplitude. We also conjecture that this remains true for higher multiplicity.

Single soft theorems and extended NLSM: In Ref. [100] it was discovered that various amplitudes contain hidden so-called extended theories in their single soft limits. For the NLSM, schematically this limit is

$$
A_{n}=z \sum_{i} s_{\mathrm{in}} A_{n-1}^{\mathrm{NLSM} \oplus \phi^{3}}(i)+\mathcal{O}\left(z^{2}\right),
$$

where $A_{n}^{\mathrm{NLSM} \oplus \phi}$ is an amplitude of pions interacting with biadjoint scalars. Its Feynman rules were found in Ref. [53], but we can derive even these mixed amplitudes simply by imposing on an appropriate Ansatz that each NLSM pion obeys the NLSM soft theorems or operators.

Direc-Born-Infeld.-DBI satisfies double soft theorems up to order $\mathcal{O}\left(z^{3}\right)$ [57], so we are looking for the lowest mass dimension objects with $\mathcal{O}\left(z^{4}\right)$ scaling in all double soft limits, which is again the Galileon vertex.

The DBI Ansatz has a form $B_{n}\left(p^{2 n-4+\kappa}\right)$ with quartic propagators, so taking into account the four momenta needed to cancel the two 3-particle poles for $n \geq 8$, we can obtain the DBI amplitudes for

$$
2 n-4+\kappa<2 n-2+4 .
$$

We conjecture the same cases can be obtained from soft operators (with the usual caveats), and that the soft Adler zero implies the $\kappa=0$ case.

But simple counting shows that an interesting stronger statement also holds: $\kappa=0 \mathrm{DBI}$ is completely fixed by just the leading and subleading theorems, and therefore the subsubleading theorem is not independent.

Dilaton.-The conformal dilaton $[40,88,89]$ obeys leading and subleading soft theorems up to order $\mathcal{O}(z)$. These encode the scaling and conformal symmetries, respectively, and are explicitly given in Refs. [42,87].

Depending on how conformal invariance is broken, two theories emerge. For spontaneous breaking, the dilaton is a Goldstone boson in the spectrum of the theory, so the amplitudes have propagators. For explicit breaking, the dilaton can be thought of as an external source, so there are no poles.

Explicit breaking: In this case we consider a polynomial Ansatz $B_{n}\left(p^{4+\kappa}\right)$, with $A^{\text {enh }}$ given by the Galileon vertex, with $[2 n-2]$. The trivially $\mathcal{O}\left(z^{2}\right)$-scaling object is $[2 n]$, so for

$$
4+\kappa<2 n-2,
$$

all amplitudes are fixed by soft theorems. 
This implies that we can obtain higher and higher corrections as we increase the multiplicity. On the other hand, the counting also implies that the subleading theorem is not independent, so scale invariance implies conformal invariance. Since we do not assume unitarity, this generalizes the result of Ref. [42].

The soft operator approach can also be checked to reproduce the dilaton amplitudes computed in Refs. [88,89]. For arbitary $n$ and $\kappa$ we conjecture that soft operators also fix amplitudes when Eq. (32) is satisfied.

Spontaneous breaking: For this case we also allow quartic poles in an Ansatz $B_{n}\left(p^{4+\kappa}\right)$, and impose the soft theorems. For $\kappa=0$ the solutions we find are identical to the explicit breaking case. For $\kappa=2$ at $n=6$, we obtain the following general solution:

$$
B_{6}\left(p^{6}\right)=a_{1} A_{6}^{\text {explicit }}\left(p^{6}\right)+a_{2} A_{6}^{\mathrm{DBI}},
$$

which is as expected, since the DBI action appears manifestly in the dilaton effective action [87]. We expect that similar behavior also holds at higher multiplicity.

Conclusions. - We have shown that imposing soft theorems completely fixes a wide range of scattering amplitudes. Further, we have conjectured that in some cases the same should hold when imposing the less constraining soft operators or soft gauge invariance or Adler zero.

For both conceptual and practical purposes, it would be extremely useful to transform these uniqueness results into a general inverse soft limit type construction [101-104]. This in turn could make manifest yet a new facet of scattering amplitudes, that of asymptotic symmetries, perhaps leading to a purely holographic description of the $S$ matrix.

The author would like to thank Nima Arkani-Hamed and Song He for discussions, comments, and collaboration on related work; John Joseph Carrasco for discussions, and Congkao Wen for discussions and comments on the manuscript. The author is grateful to the Institute of Theoretical Physics, Chinese Academy of Sciences, Beijing, where part of this work was completed. This work is supported by the European Research Council under Grant No. ERC-STG639729, "preQFT: Strategic Predictions for Quantum Field Theories."

[1] A. Strominger, J. High Energy Phys. 07 (2014) 151.

[2] A. Strominger, J. High Energy Phys. 07 (2014) 152.

[3] T. He, P. Mitra, A. P. Porfyriadis, and A. Strominger, J. High Energy Phys. 10 (2014) 112.

[4] T. He, V. Lysov, P. Mitra, and A. Strominger, J. High Energy Phys. 05 (2015) 151.

[5] D. Kapec, V. Lysov, S. Pasterski, and A. Strominger, Ann. Math. Sci. Appl. 2, 69 (2017).

[6] A. Strominger, arXiv:1703.05448.

[7] S. W. Hawking, M. J. Perry, and A. Strominger, J. High Energy Phys. 05 (2017) 161.
[8] S. W. Hawking, M. J. Perry, and A. Strominger, Phys. Rev. Lett. 116, 231301 (2016).

[9] A. Strominger, arXiv:1706.07143.

[10] F. E. Low, Phys. Rev. 110, 974 (1958).

[11] S. Weinberg, Phys. Rev. 140, B516 (1965).

[12] A. Strominger and A. Zhiboedov, J. High Energy Phys. 01 (2016) 086.

[13] F. Cachazo and A. Strominger, arXiv:1404.4091.

[14] E. Casali, J. High Energy Phys. 08 (2014) 077.

[15] C. White, Phys. Lett. B 737, 216 (2014).

[16] M. Campiglia and A. Laddha, Phys. Rev. D 90, 124028 (2014).

[17] M. Campiglia and A. Laddha, J. High Energy Phys. 12 (2015) 001.

[18] M. Campiglia and A. Laddha, J. High Energy Phys. 04 (2015) 076.

[19] M. Campiglia and A. Laddha, J. High Energy Phys. 01 (2017) 036.

[20] M. Campiglia, L. Coito, and S. Mizera, Phys. Rev. D 97, 046002 (2018).

[21] A. Laddha and P. Mitra, J. High Energy Phys. 05 (2018) 132.

[22] H. Elvang, C. R. T. Jones, and S. G. Naculich, Phys. Rev. Lett. 118, 231601 (2017).

[23] A. L. Guerrieri, Y.-t. Huang, Z.-Z. Li, and C. Wen, J. High Energy Phys. 12 (2017) 052.

[24] Y.-t. Huang, O. Schlotterer, and C. Wen, J. High Energy Phys. 09 (2016) 155.

[25] Y. Hamada and S. Sugishita, J. High Energy Phys. 11 (2017) 203.

[26] E. Conde and P. Mao, Phys. Rev. D 95, 021701 (2017).

[27] P. Mao and J.-B. Wu, Phys. Rev. D 96, 065023 (2017).

[28] P. Mao and H. Ouyang, Phys. Lett. B 774, 715 (2017).

[29] E. Conde and P. Mao, J. High Energy Phys. 05 (2017) 060.

[30] V. Lysov, arXiv:1512.03015.

[31] Z.-Z. Li, H.-H. Lin, and S.-Q. Zhang, J. High Energy Phys. 12 (2017) 032.

[32] M. Pate, A.-M. Raclariu, and A. Strominger, J. High Energy Phys. 06 (2018) 138.

[33] M. Pate, A.-M. Raclariu, and A. Strominger, Phys. Rev. Lett. 119, 261602 (2017).

[34] P. Di Vecchia, R. Marotta, and M. Mojaza, Fortschr. Phys. 64, 389 (2016).

[35] P. Di Vecchia, R. Marotta, and M. Mojaza, J. High Energy Phys. 05 (2015) 137.

[36] P. Di Vecchia, R. Marotta, M. Mojaza, and J. Nohle, Phys. Rev. D 93, 085015 (2016).

[37] P. Di Vecchia, R. Marotta, and M. Mojaza, J. High Energy Phys. 06 (2016) 054.

[38] P. Di Vecchia, R. Marotta, and M. Mojaza, J. High Energy Phys. 12 (2016) 020.

[39] P. Di Vecchia, R. Marotta, and M. Mojaza, J. High Energy Phys. 10 (2017) 017.

[40] P. Di Vecchia, R. Marotta, and M. Mojaza, J. High Energy Phys. 09 (2017) 001.

[41] M. Bianchi, S. He, Y.-t. Huang, and C. Wen, Phys. Rev. D 92, 065022 (2015).

[42] M. Bianchi, A. L. Guerrieri, Y.-T. Huang, C.-J. Lee, and C. Wen, J. High Energy Phys. 10 (2016) 036.

[43] B. U. W. Schwab, J. High Energy Phys. 08 (2014) 062. 
[44] S. Chakrabarti, S. P. Kashyap, B. Sahoo, A. Sen, and M. Verma, J. High Energy Phys. 12 (2017) 150.

[45] A. Laddha and A. Sen, J. High Energy Phys. 10 (2017) 065.

[46] A. Sen, J. High Energy Phys. 11 (2017) 123.

[47] A. Sen, J. High Energy Phys. 06 (2017) 113.

[48] Y. Hamada and G. Shiu, Phys. Rev. Lett. 120, 201601 (2018).

[49] Z.-Z. Li, H.-H. Lin, and S.-Q. Zhang, Phys. Rev. D 98, 045004 (2018).

[50] J. Broedel, M. de Leeuw, J. Plefka, and M. Rosso, Phys. Rev. D 90, 065024 (2014).

[51] Z. Bern, S. Davies, P. Di Vecchia, and J. Nohle, Phys. Rev. D 90, 084035 (2014).

[52] I. Low, Phys. Rev. D 93, 045032 (2016).

[53] I. Low and Z. Yin, Phys. Rev. Lett. 120, 061601 (2018).

[54] I. Low and Z. Yin, J. High Energy Phys. 10 (2018) 078.

[55] F. Cachazo, S. He, and E. Y. Yuan, J. High Energy Phys. 07 (2015) 149.

[56] F. Cachazo, S. He, and E. Y. Yuan, J. High Energy Phys. 07 (2014) 033.

[57] F. Cachazo, S. He, and E. Y. Yuan, Phys. Rev. D 92, 065030 (2015).

[58] F. Cachazo, S. He, and E. Y. Yuan, Phys. Rev. Lett. 113, 171601 (2014).

[59] B. U. W. Schwab and A. Volovich, Phys. Rev. Lett. 113, 101601 (2014).

[60] A. Volovich, C. Wen, and M. Zlotnikov, J. High Energy Phys. 07 (2015) 095.

[61] A. E. Lipstein, J. High Energy Phys. 06 (2015) 166.

[62] Y. Geyer, A. E. Lipstein, and L. Mason, Classical Quantum Gravity 32, 055003 (2015).

[63] C. Cheung, C.-H. Shen, and C. Wen, J. High Energy Phys. 02 (2018) 095.

[64] F. Loebbert, M. Mojaza, and J. Plefka, J. High Energy Phys. 05 (2018) 208.

[65] S. L. Adler, Phys. Rev. 137, B1022 (1965).

[66] L. Susskind and G. Frye, Phys. Rev. D 1, 1682 (1970).

[67] G. Dvali, G. Gabadadze, and M. Porrati, Phys. Lett. B 485, 208 (2000).

[68] A. Nicolis, R. Rattazzi, and E. Trincherini, Phys. Rev. D 79, 064036 (2009).

[69] K. Kampf, J. Novotný, and J. Trnka, J. High Energy Phys. 05 (2013) 032.

[70] C. Cheung, C.-H. Shen, and J. Trnka, J. High Energy Phys. 06 (2015) 118.

[71] C. Cheung, K. Kampf, J. Novotny, C.-H. Shen, and J. Trnka, J. High Energy Phys. 02 (2017) 020.

[72] C. Cheung, K. Kampf, J. Novotny, and J. Trnka, Phys. Rev. Lett. 114, 221602 (2015).

[73] C. Cheung, K. Kampf, J. Novotny, C.-H. Shen, J. Trnka, and C. Wen, Phys. Rev. Lett. 120, 261602 (2018).
[74] C. Cheung, K. Kampf, J. Novotny, C.-H. Shen, and J. Trnka, Phys. Rev. Lett. 116, 041601 (2016).

[75] H. Luo and C. Wen, J. High Energy Phys. 03 (2016) 088.

[76] H. Elvang, M. Hadjiantonis, C. R. T. Jones, and S. Paranjape, arXiv:1806.06079.

[77] N. Arkani-Hamed, F. Cachazo, and J. Kaplan, J. High Energy Phys. 09 (2010) 016.

[78] W. M. Chen, Y. tin Huang, and C. Wen, J. High Energy Phys. 03 (2015) 150.

[79] Z. Bern, J. J. Carrasco, W.-M. Chen, A. Edison, H. Johansson, J. Parra-Martinez, R. Roiban, and M. Zeng, Phys. Rev. D 98, 086021 (2018).

[80] N. Arkani-Hamed, L. Rodina, and J. Trnka, Phys. Rev. Lett. 120, 231602 (2018).

[81] L. Rodina, arXiv:1612.06342.

[82] L. Rodina, arXiv:1612.03885.

[83] R. Britto, F. Cachazo, B. Feng, and E. Witten, Phys. Rev. Lett. 94, 181602 (2005).

[84] R. Britto, F. Cachazo, and B. Feng, Nucl. Phys. B715, 499 (2005).

[85] N. Arkani-Hamed and J. Kaplan, J. High Energy Phys. 04 (2008) 076.

[86] R. H. Boels and W. Wormsbecher, arXiv:1507.08162.

[87] Y.-t. Huang and C. Wen, J. High Energy Phys. 12 (2015) 001.

[88] H. Elvang and T. M. Olson, J. High Energy Phys. 03 (2013) 034.

[89] H. Elvang, D. Z. Freedman, L.-Y. Hung, M. Kiermaier, R. C. Myers, and S. Theisen, J. High Energy Phys. 10 (2012) 011.

[90] J. J. M. Carrasco, C. R. Mafra, and O. Schlotterer, J. High Energy Phys. 06 (2017) 093.

[91] L. A. Barreiro and R. Medina, Nucl. Phys. B886, 870 (2014).

[92] L. A. Barreiro and R. Medina, J. High Energy Phys. 10 (2012) 108.

[93] S. He and Y. Zhang, J. High Energy Phys. 02 (2017) 019.

[94] K. Kampf, J. Novotný, and J. Trnka, Phys. Rev. D 87, 081701 (2013).

[95] Y.-J. Du and H. Luo, J. High Energy Phys. 08 (2015) 058.

[96] Z. Bern, J. J. M. Carrasco, and H. Johansson, Phys. Rev. D 78, 085011 (2008).

[97] G. Chen and Y.-J. Du, J. High Energy Phys. 01 (2014) 061.

[98] Y.-J. Du and C.-H. Fu, J. High Energy Phys. 09 (2016) 174.

[99] J. J. M. Carrasco, C. R. Mafra, and O. Schlotterer, J. High Energy Phys. 08 (2017) 135.

[100] F. Cachazo, P. Cha, and S. Mizera, J. High Energy Phys. 06 (2016) 170.

[101] N. Arkani-Hamed, F. Cachazo, C. Cheung, and J. Kaplan, J. High Energy Phys. 03 (2010) 020.

[102] D. Nguyen, M. Spradlin, A. Volovich, and C. Wen, J. High Energy Phys. 07 (2010) 045.

[103] C. Boucher-Veronneau and A. J. Larkoski, J. High Energy Phys. 09 (2011) 130.

[104] D. Nandan and C. Wen, J. High Energy Phys. 08 (2012) 040. 